This document is the accepted manuscript version of the following article:

Bannwarth, M. B., Camerlo, A., Ulrich, S., Jakob, G., Fortunato, G., Rossi, R. M., \& Boese1, L. F. (2015). Ellipsoid-shaped superparamagnetic nanoclusters through emulsion

electrospinning. Chemical Communications, 51(18), 3758-3761. https://doi.org/10.1039/C4CC10076J

\title{
COMMUNICATION
}

Cite this: DOI: $10.1039 / \times 0 \times x 00000 x$

\section{Ellipsoid-Shaped Superparamagnetic Nanoclusters through Emulsion Electrospinning}

\author{
Markus B. Bannwarth ${ }^{a^{*}}$, Agathe Camerlo ${ }^{a}$, Sebastian Ulrich ${ }^{\mathrm{a}}$, Gerhard Jakob ${ }^{\mathrm{b}}$, \\ Giuseppino Fortunato ${ }^{\mathrm{a}}$, René M. Rossi ${ }^{\mathrm{a}}$, Luciano F. Boesel ${ }^{\mathrm{a}^{*}}$
}

Received 00th January 2012,

Accepted 00th January 2012

DOI: $10.1039 / x 0 x \times 00000 x$

www.rsc.org/

\begin{abstract}
Ellipsoid-shaped nanoclusters composed of single superparamagnetic nanoparticles can be generated by emulsion electrospinning. Stretching and subsequent solvent evaporation of iron oxide loaded emulsion droplets during the emulsion electrospinning process enables the creation of such structures embedded in polymer nanofibers. Dissolution of the polymer fibers yields an aqueous dispersion of the inorganic clusters which are the first example of ellipsoid-shaped superparamagnetic nanoclusters with a high saturation magnetization $\left(\sim 47 \mathrm{emu} \cdot \mathrm{g}^{-1}\right)$.
\end{abstract}

Superparamagnetic nanoparticles have drawn tremendous attention in the field of biomedical applications. ${ }^{1}$ Thanks to their strong response to external magnetic fields, they are powerful contrast enhancers for magnetic resonance imaging (MRI), 3 and heat sources for hyperthermia ${ }^{4}$ or triggered drug release. ${ }^{5}$ The superparamagnetic effect is strongly size-limited and only exists for iron oxide nanoparticles that are smaller than about $20 \mathrm{~nm}^{6}$ However, superparamagnetic particles can be clustered to preserve and even improve their properties. ${ }^{7,8}$ Implementing them as contrast agents for MRI, their nature to shorten the transverse relaxation of water is enhanced, since they collectively act as nanoparticle ensemble. ${ }^{9}$ Today, many examples for spherical superparamagnetic nanoparticle clusters are explained in literature. ${ }^{10}$ However their ellipsoid analogues are found very rarely, even if shape anisotropy is considered to impose a very strong influence on the magnetization properties. ${ }^{11}$ Anisotropic magnetic materials are believed to possess great potential in magnetomechanical systems, high density data storage systems or sensing applications. ${ }^{12}$ Additionally, shape plays a major role for nanoparticle-cell interactions ${ }^{13,14}$ or for nanoparticle drying/patterning on surfaces. ${ }^{15}$ Besides assembling superparamagnetic nanoclusters into linear chains to create onedimensionality (1D), ${ }^{16-18}$ Nanoparticles with ellipsoid shape are accessible as hematites and goethites. ${ }^{19-21}$ However, the described iron oxide based rices/ellipsoids either reach a rather low saturation magnetization of $\sim 10 \mathrm{emu} \cdot \mathrm{g}^{-1}$ or lower (and between $2-26 \mathrm{emu} \cdot \mathrm{g}^{-1}$ for different $\mathrm{Mn}-$, Ni-, Cu-, Sn-doped samples) and/or show a significant remanence. Since superparamagnetic iron oxides can reach values of $>90 \mathrm{emu} \cdot \mathrm{g}^{-1},{ }^{22}$ the saturation magnetization of ellipsoidshape superparamagnetic nanoparticle clusters can still be strongly improved and novel methods for their preparation are required. ${ }^{23}$

Hence, the question arises whether there is a possibility to cluster single superparamagnetic nanocrystals permanently in an ellipsoid shape. Indeed, this becomes possible when making use of an elongation-evaporation process that can be applied via electrospinning to an aqueous emulsion containing octane droplets with a high loading of superparamagnetic nanoparticles (Figure 1). The process is inspired by the elongation of solvent-swollen polymer nanoparticles during the electrospinning process. ${ }^{24}$ For axial deformation of initially spherical to elongated droplets, it is mandatory to apply a unidirectional stress on the droplet. It is well known that during the electrospinning process stresses of 10-100 $\mathrm{kPa}$ are present. ${ }^{25}$ Although it is reported that during emulsion electrospinning the droplets can become deformed in the jet whipping area where rapid jet thinning and thus, stress generation occurs, ${ }^{26}$ this stretching phenomenon has never been used to make elongated inorganic materials. The possibility to generate ellipsoid-shaped superparamagnetic iron oxide nanoclusters (ESIONCs) via emulsion electrospinning is depicted in Figure 1. Firstly, easily accessible oleate-capped iron oxide nanoparticles with superparamagnetic properties were redispersed in high solid contents (50 wt.\%) in $n$ octane. Octane is very suitable for the redispersion of the oleatecapped iron oxide nanoparticles. ${ }^{27}$ By addition of this dispersion to a continuous aqueous phase containing sodium dodecylsulfate (SDS) as surfactant and application of ultrasonic treatment, a miniemulsion of octane nanodroplets with a high content of iron oxide nanoparticles is formed. This miniemulsion is mixed with an aqueous poly (vinyl 
alcohol) (PVA) solution to yield in a miniemulsion containing $10 \mathrm{wt} . \%$ of PVA. The viscous miniemulsion is then inserted in the electrospinning process. During electrospinning, the elongationevaporation process occurs. Here, the continuous PVA/water phase is spun to form nanofibers while the disperse phase of superparamagnetic octane droplets is embedded in the PVA fibers. For the generation of the ESIONCs, thinning of the jet and subsequent evaporation of water leads to a squeezing of the magnetic droplets in direction of the fiber axis. Then, the octane, with its higher boiling point than water $\left(\sim 125{ }^{\circ} \mathrm{C}\right)$, evaporates, and solid ESIONCs are formed from the elongated liquid droplets. When we replaced the octane and used chloroform instead under similar conditions, we could not observe elongated clusters of iron oxide nanoparticles. Instead, spherical clusters were found. Hence, the solvent cannot easily be exchanged.

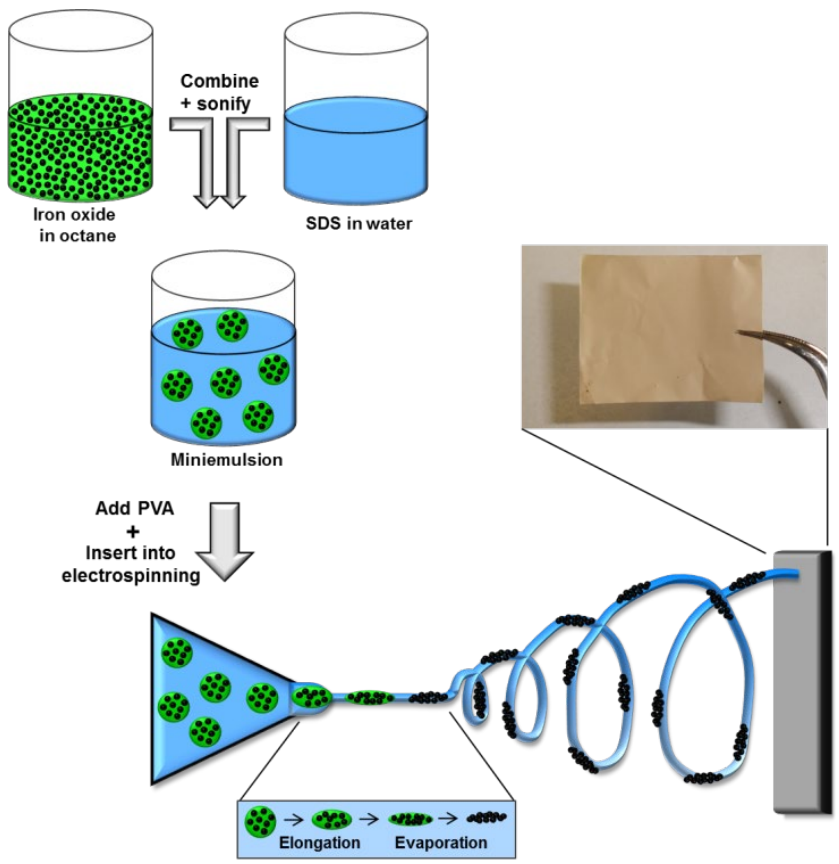

Fig. 1 Schematic illustration of the fabrication of ESIONCs. An octane phase containing iron oxide nanoparticles is mixed with a SDScontaining aqueous solution and sonified to form a direct miniemulsion. After addition of PVA to the miniemulsion, electrospinning is performed. During the electrospinning process, the ESIONCs are formed by elongation and evaporation of the octane droplets. Finally, the ESIONCs are found embedded in the fibrous PVA mesh. Top right: Optical micrograph of a brown-colored electrospun iron oxide/PVA mesh on an aluminum foil.

Investigation of the morphology of the formed fibers and the structure of the incorporated ESIONCs was done with electron microscopy (Figure 2). SEM and STEM overview images show a mesh of nanofibers (Figure $2 \mathrm{a}+\mathrm{b}$ ). A closer look at the interior structure of the fibers reveals an elongated cluster consisting of many single nanoparticles as it is embedded in the fibrous PVA network (Figure 2c).

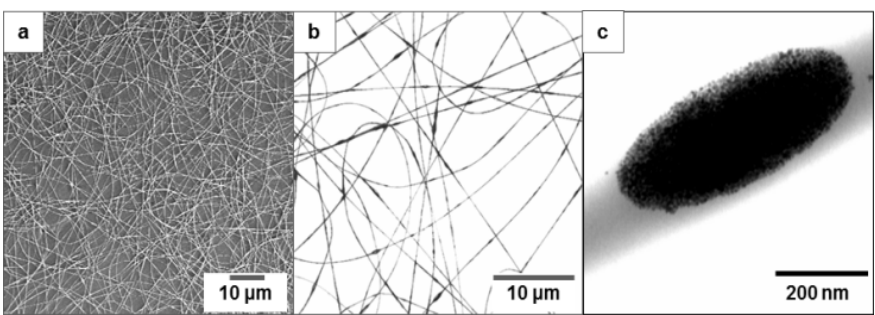

Fig. 2 SEM (a) and STEM (b, c) micrographs of electrospun PVA fibers with incorporated ESIONCs.

Importantly, the elongation of the iron oxide clusters is related to the final fiber diameter. From STEM images, one can clearly see that with a decreasing diameter of the PVA fiber, the elongation of the clusters increases considerably (Figure 3a-f). The elongation, however, is not strongly influenced by the size of the nanoparticle cluster (Average cluster diameter: Equatorial axis: $94 \pm 40 \mathrm{~nm}$; Polar axis: $250 \pm 114 \mathrm{~nm}$ ). Similar aspect ratios are obtained for differently sized clusters, whenever the fiber has the same diameter. This phenomenon can be observed especially in the Figures $3 \mathrm{~d}+\mathrm{e}$, where differently sized clusters are embedded in the same fiber and show similar stretching behavior.

In order to quantify the relationship between the cluster elongation and the fiber diameter, several fibers with different diameters were analyzed and the aspect ratio of the ESIONCs is plotted against the fiber diameter (Figure $3 \mathrm{~g}$ ). The ESIONCs were analyzed from a mesh of nanofibers with various fiber diameters $(161 \pm 58 \mathrm{~nm})$. Hence, the aspect ratio of the ESIONCs can directly be correlated to the fiber diameter (for a given PVA concentration of 10 wt.\%). For fiber diameters above $200 \mathrm{~nm}$, the clusters are only weakly elongated and the aspect ratio lies near 1 . When the fiber diameter is lower than 200 $\mathrm{nm}$, efficient stretching is achieved. For the extreme case when the fiber diameter is close to $50 \mathrm{~nm}$, the aspect ratio can be very high, reaching a value of nearly 10 . In principle, the fiber diameter is influenced by many electrospinning conditions. ${ }^{28-30}$ In order to evaluate the effect on the fiber diameter and the stretching of the clusters, we investigated some of these parameters. When varying the applied voltage (6-12 V) and the distance between tip and collector $(15-25 \mathrm{~cm})$, we observed no significant change in the fiber diameter and the aspect ratio of the ESIONCs. In contrast, a variation of the PVA concentration affects the elongation substantially. When the PVA content is too low, no stretching is observed, resulting in spherical iron oxide clusters. Reducing the PVA concentration, the stretching is reduced (Figure S2c) and below 7 wt.\% hardly any stretching of the clusters was observed anymore (Figure S2a, b). >10 wt.\% PVA concentrations can be used to obtain stretched clusters. However, the viscosity significant increases and a homogeneous mixing of the iron oxide/octane emulsion with the PVA solution becomes difficult. Hence, a PVA concentration of $\sim 10 \mathrm{wt} . \%$ is well suited to make ESIONCs with high aspect ratio. The high dependency of the stretching of iron oxide clusters with PVA concentration can be explained by the strong viscosity increase of aqueous solutions with increasing PVA concentration (Figure S3). With an increasing viscosity of the continuous phase, the emulsion droplets tend to 
accompany the elongation of the fiber instead of maintaining their spherical shape.

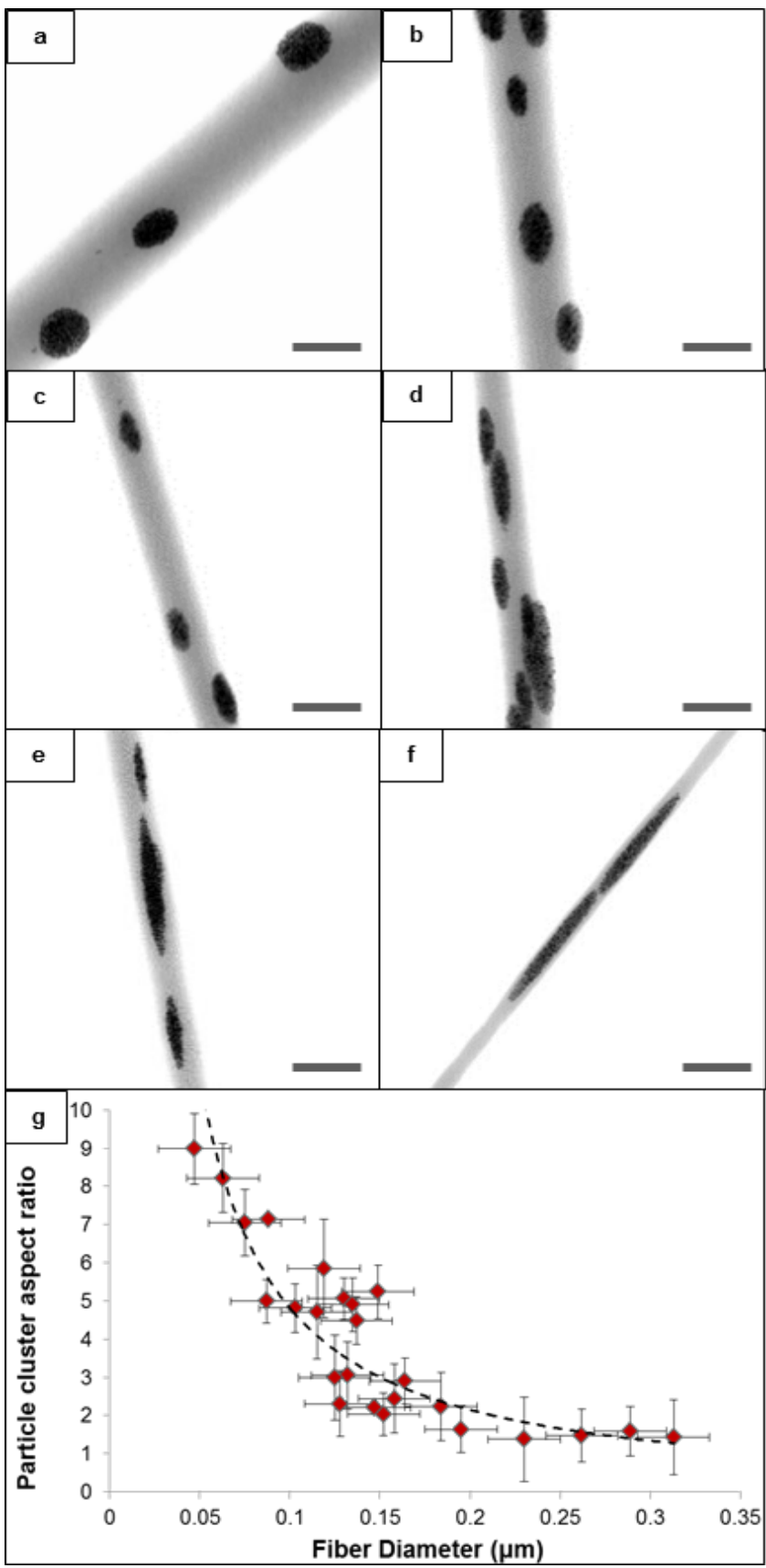

Fig. 3 STEM images of ESIONCs incorporated in electrospun PVA fibers obtained from a 10 wt.\% PVA emulsion. With decreasing diameter of the fibers, the aspect ratio of the ESIONCs increases considerably (from a-f). The elongation of the iron oxide clusters occurs mainly independent of the size of the cluster yielding in similar aspect ratio of differently sized particles within the same fiber (d, e). Scale bar: $200 \mathrm{~nm}$. g) Relationship between the aspect ratio of the ESIONCs and the fiber diameter. With a decreasing fiber diameter, the aspect ratio of the ESIONCs increases considerably.

Since the ESIONCs are embedded in a fiber mesh of PVA, the fibers can easily be dissolved in water (see experimental section) and the
ESIONCs acquired as an aqueous dispersion (Figure 4a-c). The dispersion shows no significant change in size and polydispersity in a $\mathrm{pH}$ range between 3 and 10 as measured by DLS. The mechanical stability of the ESIONCs was tested by putting the ESIONC dispersion in an ultrasonication bath for $1 \mathrm{~min}$. After such treatment one can observe a slight deformation and degradation of the ESIONCs via STEM imaging (Figure S5), revealing a vulnerability of the ESIONCs towards ultrasonic treatment. The ESIONCs contain 67 wt.\% of iron oxide as determined via thermogravimetric analysis (TGA). The crystal structure was determined from an X-ray diffraction (XRD) pattern and found to be consistent with the structure of magnetite (Figure 4f). The magnetic properties of the ESIONCs were analyzed via a Superconducting Quantum Interference Device (SQUID) magnetometer. The measurements reveal a high saturation magnetization value of the ESIONCs $\left(\sim 47 \mathrm{emu} \cdot \mathrm{g}^{-1}\right.$ for the clusters themselves and $\sim 70 \mathrm{emu} \cdot \mathrm{g}^{-1}$ for the iron oxide part), which is similar to the one of the single oleate-capped iron oxide nanoparticles (Figure S6) and a blocking temperature of around $150 \mathrm{~K}$ (Figure 4d, e). Hence, the clusters are the first example of ellipsoid-shaped iron oxide nanoclusters with a high saturation magnetization.

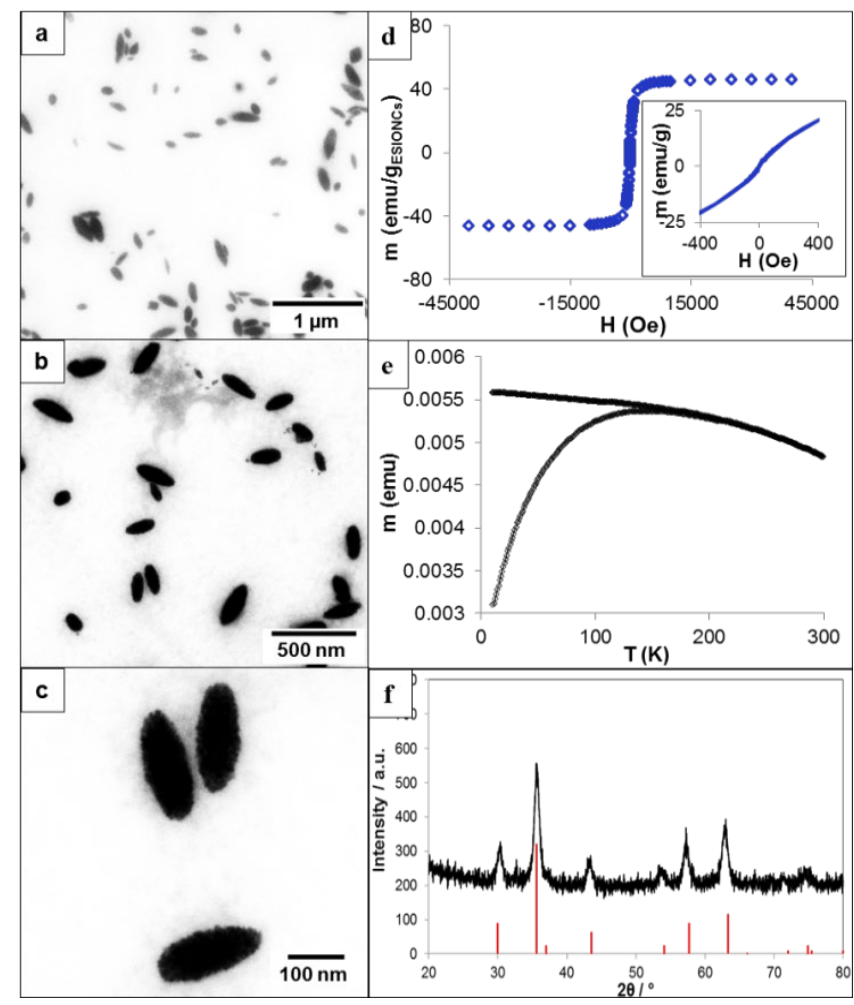

Fig. 4 STEM images of ESIONCs at different magnifications (a-c). The ESIONCs were obtained after dissolving the PVA nanofibers and redispersion of the ESIONCs in water. d) SQUID magnetization curve of ESIONCs at $300 \mathrm{~K}$ (with an inset showing no visible hysteresis). e) Temperature dependent magnetization of ESIONCs. f) XRDpattern of the iron oxide nanoparticles (red peaks correspond to magnetite).

In summary, we have demonstrated for the first time the accumulation of single superparamagnetic iron oxide nanoparticles into ellipsoid-shape nanoclusters with 
superparamagnetic properties and a high saturation magnetization. The elongated clusters of iron oxides are generated via emulsion electrospinning following an elongationsolvent evaporation mechanism. A strong correlation between the aspect ratio of the obtained ESIONCs and the fiber diameter was found. After elongation, the elongated clusters are embedded in a PVA matrix, which can easily be dissolved with water to yield an aqueous dispersion of the ESIONCs suitable for biomedical applications.

The authors thank Dr. Songhak Yoon for XRD measurements, Manel Beldi for rheology measurements and Lea Bommer for TGA analysis.

\section{Notes and references}

${ }^{a}$ Empa, Swiss Federal Laboratories for Materials Science and Technology, Laboratory for Protection and Physiology, Lerchenfeldstrasse 5, CH-9014 St. Gallen, Switzerland.

${ }^{b}$ Institute of Physics, University of Mainz, Staudingerweg 7, 55128 Mainz, Germany.

Electronic Supplementary Information (ESI) available:

1. Neuberger, T.; Schöpf, B.; Hofmann, H.; Hofmann, M.; von Rechenberg, B., J. Magn. Magn. Mater. 2005, 293, 483-496.

2. Amstad, E.; Zurcher, S.; Mashaghi, A.; Wong, J. Y.; Textor, M.; Reimhult, E., Small 2009, 5, 1334-1342.

3. Hu, F.; MacRenaris, K. W.; A. Waters, E.; Schultz-Sikma, E. A.; Eckermann, A. L.; Meade, T. J., Chem. Commun. 2010, 46, 73-75.

4. Jordan, A.; Scholz, R.; Wust, P.; Fahling, H.; Felix, R., J. Magn. Magn. Mater. 1999, 201, 413-419.

5. Bannwarth, M. B.; Ebert, S.; Lauck, M.; Ziener, U.; Tomcin, S.; Jakob, G.; Münnemann, K.; Mailänder, V.; Musyanovych, A.; Landfester, K., Macromolecular Bioscience 2014, 14, 1205-1214.

6. Teja, A. S.; Koh, P. Y., Prog. Cryst. Growth Charact. Mater. 2009, 55, $22-45$.

7. Berret, J.-F.; Schonbeck, N.; Gazeau, F.; El Kharrat, D.; Sandre, O.; Vacher, A.; Airiau, M., J. Am. Chem. Soc. 2006, 128, 1755-1761.

8. Larsen, B. A.; Haag, M. A.; Serkova, N. J.; Shroyer, K. R.; Stoldt, C. R., Nanotechnology 2008, 19.

9. Roch, A.; Gossuin, Y.; Muller, R. N.; Gillis, P., J. Magn. Magn. Mater. 2005, 293, 532-539.

10. Ge, J.; Hu, Y.; Biasini, M.; Beyermann, W. P.; Yin, Y., Angew. Chem. Int. Ed. 2007, 46, 4342-4345.

11. Leslie-Pelecky, D. L.; Rieke, R. D., Chem. Mater. 1996, 8, 1770-1783. 12. Yuan, J. Y.; Xu, Y. Y.; Muller, A. H. E., Chem. Soc. Rev. 2011, 40, 640-655.

13. Florez, L.; Herrmann, C.; Cramer, J. M.; Hauser, C. P.; Koynov, K.; Landfester, K.; Crespy, D.; Mailänder, V., Small 2012, 8, 2222-2230.

14. Krug, H. F.; Wick, P., Angew. Chem. Int. Ed. 2011, 50, 1260-1278.

15. Yunker, P. J.; Still, T.; Lohr, M. A.; Yodh, A. G., Nature 2011, 476, 308-311.

16. Bannwarth, M. B.; Kazer, S. W.; Ulrich, S.; Glasser, G.; Crespy, D.; Landfester, K., Angew. Chem. Int. Ed. 2013, 52, 10107-10111.

17. Bannwarth, M.; Crespy, D., Chemistry - An Asian Journal 2014, 9, 2030-2035.
18. Bannwarth, M. B.; Weidner, T.; Eidmann, E.; Landfester, K.; Crespy, D., Chem. Mater. 2014, 26, 1300-1302.

19. Rebolledo, A. F.; Bomatí-Miguel, O.; Marco, J. F.; Tartaj, P., Adv. Mater. 2008, 20, 1760-1765.

20. Rebolledo, A. F.; Laurent, S.; Calero, M.; Villanueva, A.; Knobel, M.; Marco, J. F.; Tartaj, P., ACS Nano 2010, 4, 2095-2103.

21. Li, L.; Qin, D.; Yang, X.; Liu, G., Polymer Chemistry 2010, 1, $289-$ 295.

22. Rebodos, R. L.; Vikesland, P. J., Langmuir 2010, 26, 16745-16753.

23. Sanchez-Ferrer, A.; Reufer, M.; Mezzenga, R.; Schurtenberger, P.; Dietsch, H., Nanotechnology 2010, 21.

24. Herrmann, C.; Turshatov, A.; Crespy, D., ACS Macro Letters 2012, 1, 907-909.

25. Han, T.; Yarin, A. L.; Reneker, D. H., Polymer 2008, 49, 1651-1658. 26. Sy, J. C.; Klemm, A. S.; Shastri, V. P., Adv. Mater. 2009, 21, 18141819.

27. Hofmann, D.; Tenzer, S.; Bannwarth, M. B.; Messerschmidt, C.; Glaser, S.-F.; Schild, H.; Landfester, K.; Mailänder, V., ACS Nano 2014, 8, 10077-10088.

28. Camerlo, A.; Bühlmann-Popa, A. M.; Vebert-Nardin, C.; Rossi, R.; Fortunato, G., Journal of Materials Science 2014, 49, 8154-8162.

29. Hardick, O.; Stevens, B.; Bracewell, D. G., Journal of Materials Science 2011, 46, 3890-3898.

30. Pelipenko, J.; Kristl, J.; Janković, B.; Baumgartner, S.; Kocbek, P., Int. J. Pharm. 2013, 456, 125-134. 


\section{Supporting Information}

\section{Superparamagnetic Ellipsoid Nanoclusters from Emulsion Electrospinning}

Markus B. Bannwarth ${ }^{\dot{H}^{*}}$, Agathe Camerlo ${ }^{\dagger}$,Sebastian Ulrich ${ }^{a}$, Gerhard Jakob ${ }^{\dagger}$, Giuseppino Fortunato $^{\dagger}$, René M. Rossit, Luciano F. Boesel ${ }^{t^{*}}$

†Empa, Laboratory for Protection and Physiology, Lerchenfeldstrasse 5, CH-9014 St. Gallen, Switzerland.

¥Institute of Physics, University of Mainz, Staudingerweg 7, 55128 Mainz, Germany. 


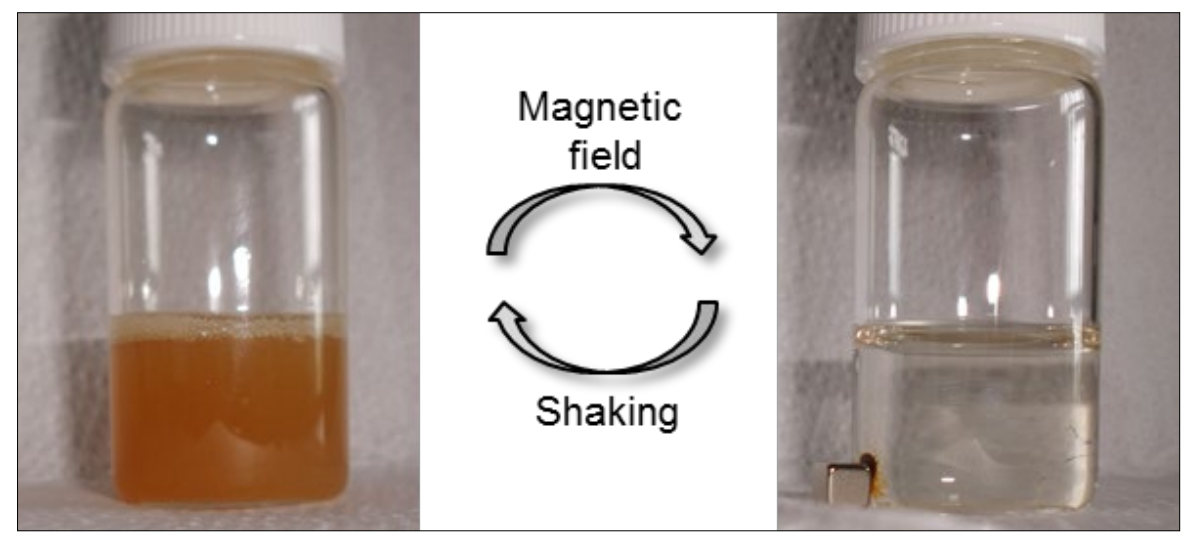

Figure S1. Optical micrograph showing the response of ESIONCs upon application of an external magnetic field applied by a small permanent magnet. When putting the permanent magnet next to a dispersion of ESIONCs, they can be easily collected next to the magnet due to their high saturation magnetization when applying the external magnetic field. 


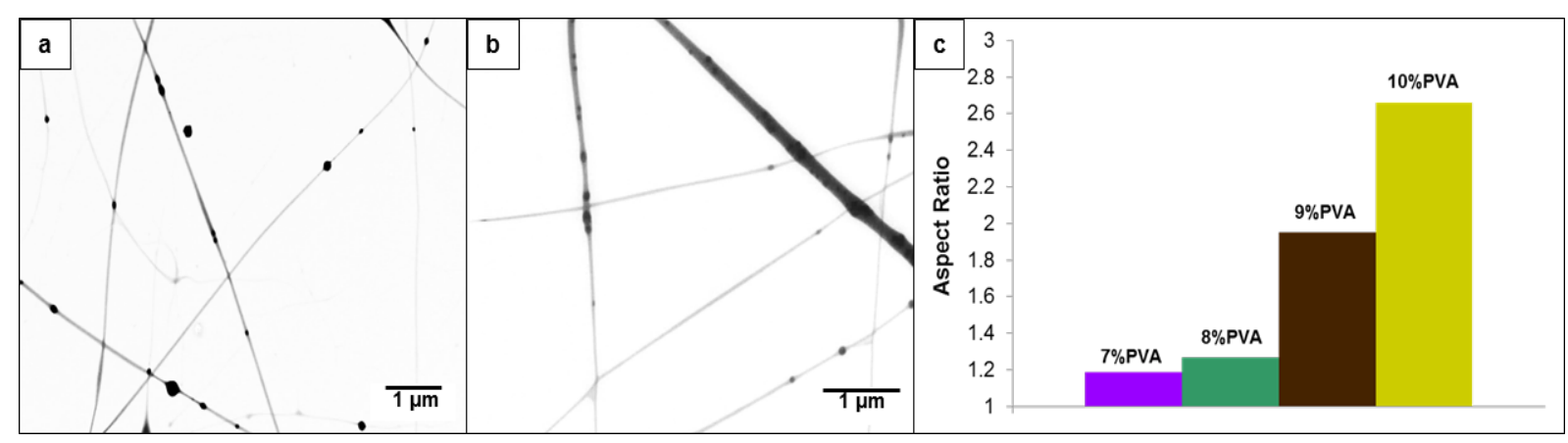

Figure S2. STEM images of fibers containing iron oxide nanoclusters. The fibers were formed from aqueous emulsions with different PVA concentrations: 3 and $7 w t \%$ in Figure a and b, respectively. Figure $c$ shows the dependency of the average aspect ratio with the PVA concentration 


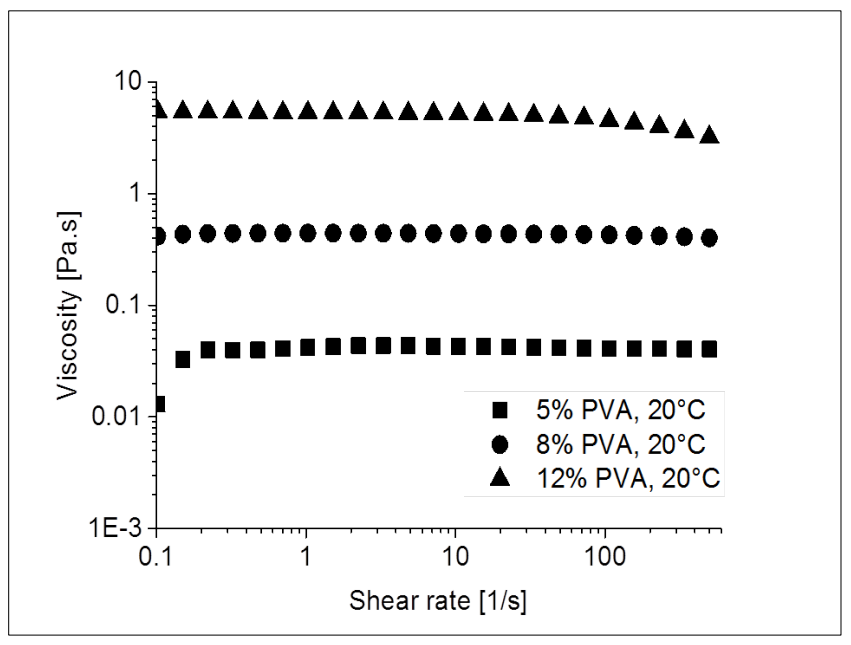

Figure S3. Viscosity of aqueous PVA solutions of 5, 8, and 12 wt.\% at different shear rates. 


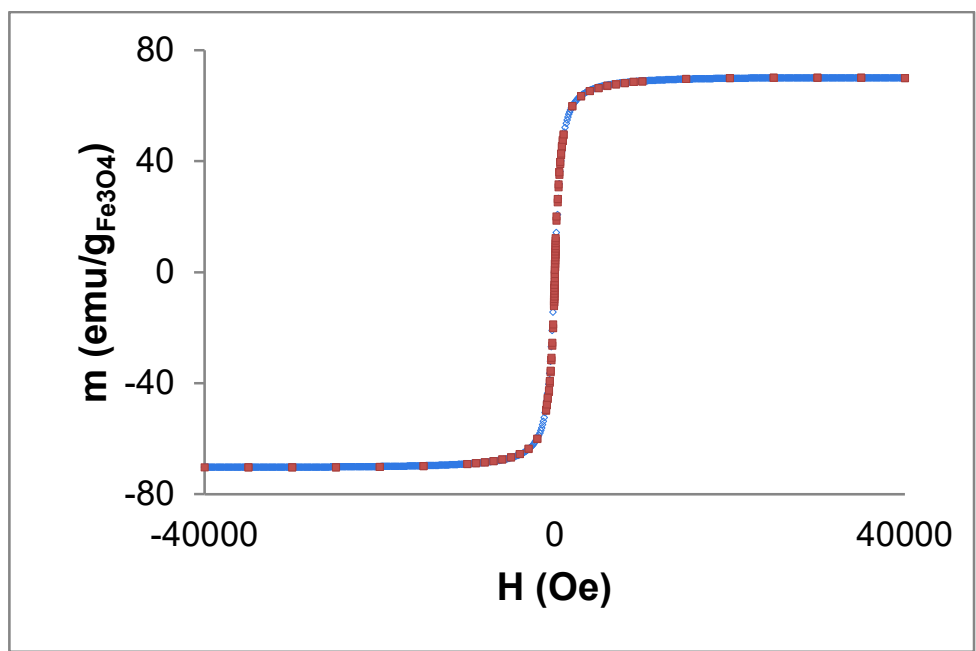

Figure S4. SQUID magnetization curve of ESIONCs as experimentally measured ( $\square$ ) and theoretically calculated $(\diamond)$. 


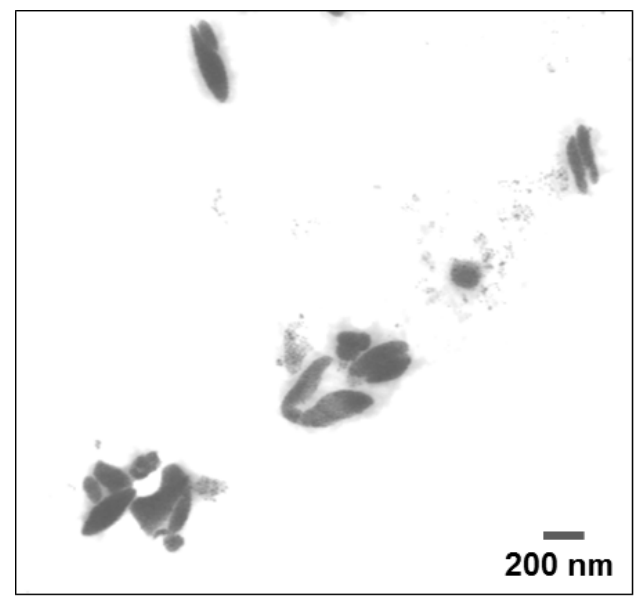

Figure S5. STEM image of ESIONCs that were treated as aqueous dispersion for 1 min in an ultrasonication bath. Most of the ESIONCs maintain their ellipsoid shape. However, one can observe the beginning of a degradation/decomposition process of the ESIONCs. 


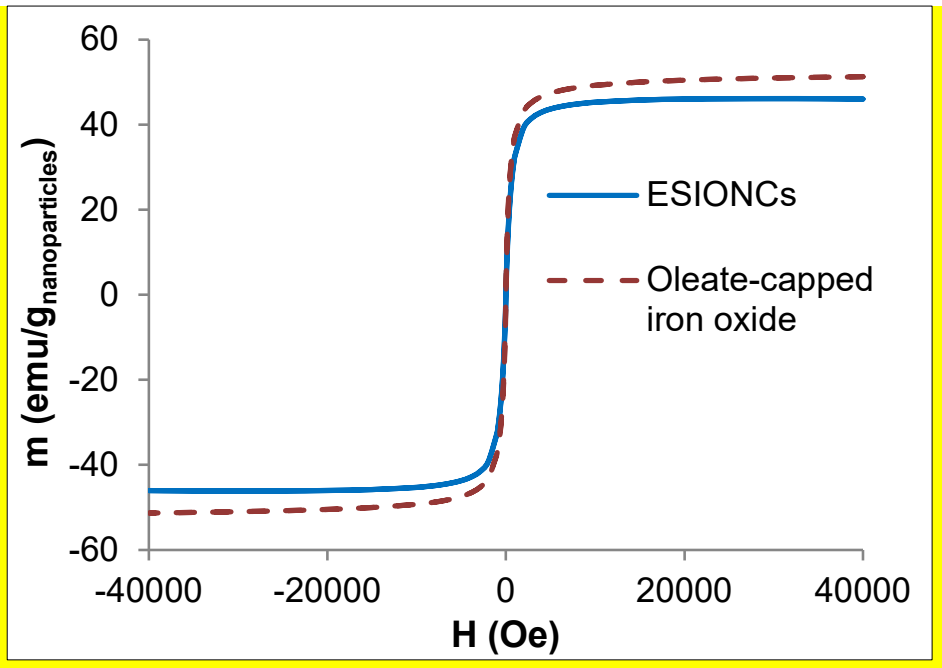

Figure S6. VSM curves of the spherical oleate-capped iron oxide nanoparticles before electrospinning and the ESIONCs. Both curves follow the same trend, showing no observable remanence. The saturation magnetization of the original, spherical oleate-capped iron oxides is slightly higher in comparison to the magnetization of the ESIONCs. This small difference can be explained by the PVA layer which sticks to the ESIONCs after electrospinning in the PVA solution. The PVA reduces the percentage of superparamagnetic iron oxides per particle and hence the saturation magnetization per gram of particles. 


\section{COMMUNICATION}

\section{Experimental}

\section{Methods}

SEM and STEM were performed on a Hitachi S-4800 (Hitachi High technologies, Canada). SEM samples were drop-casted on a silicon wafer and sputtered with gold (Polaron Equipment, SEM coating Unit E5100, Kontron AG, Switzerland, $5 \mathrm{~nm}$ thick coating). STEM samples were drop-casted on a 300 mesh carbon coated cupper grid. Solid contents were calculated from gravimetric data.

X-ray powder diffraction (XRD) patterns were obtained using a PANalytical X'Pert PRO $\theta-2 \theta$ scan system equipped with a Johansson monochromator $\left(\mathrm{Cu}-\mathrm{K} \alpha_{1}\right.$ radiation, $\left.1.5406 \AA\right)$ and an $\mathrm{X}^{\prime}$ Celerator linear detector. The diffraction pattern was recorded between $20^{\circ}$ and $80^{\circ}(2 \theta)$ with an angular step interval of $0.0167^{\circ}$.

Magnetic properties of the ESIONCs were determined via a Superconducting Quantum Interference Device (SQUID) magnetometer (Quantum Design MPMS XL). The sample material was measured in a gelatin capsule, which provides a negligible purely diamagnetic background signal. The corresponding data is depicted in Figure S4. The simulated magnetization curve in Figure S4 results from the superparamagnetic response of $\mathrm{Fe}_{3} \mathrm{O}_{4}$ particles with a total spin momentum of $\mathrm{J}=\mathrm{S}=6900$ and a diamagnetic contribution of $\chi_{\mathrm{dia}}=-1.942 \cdot 10^{-5} \mathrm{emu} \cdot(\mathrm{g} \cdot \mathrm{Oe})^{-1}$ from the sample holder and organic shell material. As magnetite provides $4 \mu_{B} \cdot$ f.u. ${ }^{-1}$ (Bohr magnetons per formula unit), approximately 3450 f.u. are contained in each nanoparticle corresponding to a diameter of a spherical nanoparticle of $7.85 \mathrm{~nm}$. Since the simulated curve stems from a unique small particle size, it is obvious that the clustered ellipsoids show the magnetic response of the individual contributing nanoparticles in this hysteresis curve. Nevertheless, shape anisotropy due to the ellipsoidal clusters can lead to additional demagnetization effects not present in the simulation. Also the outermost shell of a magnetic nanoparticle shows usually a reduced magnetic response due to the different chemical surrounding. Thus, the particle size calculated from the total spin moment is a lower boundary of the true value. On the other hand, the high value measured for the saturation magnetization limits the thickness of a nonmagnetic shell to smaller than $0.3 \mathrm{~nm}$, i.e. less than the length of a cube containing one formula unit. For the latter estimation we compared to the temperature dependence of the saturation magnetization as measured in bulk single crystals. ${ }^{1}$ The diameter of the superparamagnetic particles can therefore be estimated to be around $8.5 \mathrm{~nm}$ (core + weak magnetic outer shell), which is roughly consistent with the average crystallite size of $\sim 10 \mathrm{~nm}$ as determined from XRD measurements by using the Scherer equation.

For determination of the blocking temperature, the sample was cooled to $10 \mathrm{~K}$. A field of $150 \mathrm{Oe}$ was applied and measurements were taken during warming to $300 \mathrm{~K}$ with a rate of $2.5 \mathrm{~K} \cdot \mathrm{min}^{-1}(\mathrm{zfc})$. 
Afterwards, measurements were taken during cooling to $10 \mathrm{~K}$ in the applied field (fc). Finally the data was measured during warming without changing the magnetic field (fw). The field warming and field cooling curves are practically identical and form the upper branch of Figure 4e, while the zero field curve is the lower branch.

TGA analysis was performed on a NETZSCH TG $209 \mathrm{~F} 1$ instrument under nitrogen atmosphere from $25-800{ }^{\circ} \mathrm{C}$ with a heating rate of $10 \mathrm{~K} \cdot \mathrm{min}^{-1}$. A rheometer (Anton Paar Physica MCR 300, Austria) equipped with a plate - cone geometry was applied in controlled shear rate mode to assess the shear viscosities as a function of shear rate. Flow curves with shear rates varying from $0.01-500 \mathrm{~s}^{-1}$ were recorded at $20{ }^{\circ} \mathrm{C}$.

\section{Synthesis of Oleate-Capped Iron Oxide Nanoparticles}

All commercially available reagents and solvents were used without further purification. The synthesis of oleate-capped iron oxide nanoparticles was done as similar reported in literature. ${ }^{2,3}$ Briefly, a 90 mmol $\mathrm{FeCl}_{3} \cdot 6 \mathrm{H}_{2} \mathrm{O}$ and 60 mmol $\mathrm{FeCl}_{2} \cdot 4 \mathrm{H}_{2} \mathrm{O}$ were dissolved in $100 \mathrm{~mL}$ of demineralized water. $40 \mathrm{~mL}$ of an ammonium hydroxide solution $\left(28 \% \mathrm{NH}_{3}\right.$ in water) were added dropwise before adding 15 mmol oleic acid. Under vigorous stirring, the reaction was kept at $70{ }^{\circ} \mathrm{C}$ for $1 \mathrm{~h}$ and at $100{ }^{\circ} \mathrm{C}$ for $2 \mathrm{~h}$ while constantly refilling the evaporating water with deionized water. After complete evaporation of excess $\mathrm{NH}_{3}$, the reaction mixture was cooled to room temperature and put on top of a permanent magnet to precipitate the magnetic nanoparticles. The particles were washed several times with deionized water and dried under vacuum before further use.

\section{Preparation of Miniemulsion Droplets Containing Iron Oxide Nanoparticles}

For the formation of iron oxide containing miniemulsion droplets, $4 \mathrm{~g}$ of oleate-capped iron oxide nanoparticles were redispersed in $4 \mathrm{~g}$ of $n$-octane in an ultrasonication bath for $1 \mathrm{~h}$. The resulting black dispersion was mixed with $24 \mathrm{~g}$ of water containing $24 \mathrm{mg}$ of SDS and stirred for $1 \mathrm{~h}$ at $1400 \mathrm{rpm}$ to form a macro emulsion. Miniemulsion droplets were formed by ultrasonication under ice cooling using a Branson Digital Sonifier with $1 / 2$ inch ultrasonication tip ( $3 \mathrm{~min}, 50 \%$ amplitude, $10 \mathrm{~s}$ pulse, $5 \mathrm{~s}$ pause).

\section{Emulsion Electrospinning of the Iron Oxide Containing Miniemulsion}

The iron oxide containing miniemulsion was mixed non-magnetically over night with an aqueous solution containing 15 wt.\% Poly (vinyl alcohol) $\left(\mathrm{Mw}=205.000 \mathrm{~g} \cdot \mathrm{mol}^{-1}\right)$ to form a $10 \mathrm{wt} . \%$ PVA miniemulsion and filled into a $1 \mathrm{~mL}$ syringe. When bubbles were present in the syringe, they were removed by putting the syringe in an ultrasonication bath. Emulsion electrospinning was then performed via a previously described electrospinning setup, ${ }^{4,5}$ which was placed in a climate chamber to have good control over the spinning conditions. The emulsion was then spun at $24{ }^{\circ} \mathrm{C}$ and at a relative humidity of $60 \%(20 \mathrm{~cm}$ 
distance, $7 \mu \mathrm{L} \cdot \mathrm{min}^{-1}$ feed rate, $0.8 \mathrm{~mm}$ tip diameter, +12 and $-5 \mathrm{~V}$ and $0.1 \mathrm{~mA}$ ) and the fibers collected on top of an aluminum foil or on a carbon coated cupper grid. The fibers were analyzed via SEM and STEM.

\section{Dissolution of PVA Fibers and Subsequent Dispersion of Elongated Iron Oxide Nanoclusters}

For dissolution of the elongated nanoparticles, the fiber mesh was removed from the aluminum foil and demineralized water added. After shaking for $5 \mathrm{~min}$, the magnetic dispersion was put on top of a permanent magnet until most of the particles settled on the magnet. The supernatant was discarded and refilled with deionized water. Then the particles were analyzed by SEM, STEM, SQUID magnetometry and TGA.

1. Özdemir, Ö., Coercive force of single crystals of magnetite at low temperatures. Geophysical Journal International 2000, 141, 351-356.

2. Bannwarth, M. B.; Kazer, S. W.; Ulrich, S.; Glasser, G.; Crespy, D.; Landfester, K., Well-Defined Nanofibers with Tunable Morphology from Spherical Colloidal Building Blocks. Angew. Chem. Int. Ed. 2013, 52, 10107-10111.

3. Bannwarth, M. B.; Weidner, T.; Eidmann, E.; Landfester, K.; Crespy, D., Reversible Redox-Responsive Assembly/Disassembly of Nanoparticles Mediated by Metal Complex Formation. Chem. Mater. 2014, 26, 13001302.

4. Camerlo, A.; Vebert-Nardin, C.; Rossi, R. M.; Popa, A. M., Fragrance encapsulation in polymeric matrices by emulsion electrospinning. Eur. Polym. J. 2013, 49, 3806-3813.

5. Fortunato, G.; Guex, A. G.; Popa, A. M.; Rossi, R. M.; Hufenus, R., Molecular weight driven structure formation of PEG based e-spun polymer blend fibres. Polymer 2014, 55, 3139-3148. 